\title{
VERTICAL ACOUSTIC CONFINEMENT FOR HIGH-Q FULLY-DIFFERENTIAL CMOS-RBTS
}

\author{
Bichoy Bahr ${ }^{1^{*}}$ and Dana Weinstein ${ }^{2}$
}

${ }^{1}$ Massachusetts Institute of Technology, Cambridge, Massachusetts, USA

${ }^{2}$ Purdue University, West Lafayette, Indiana, USA

\begin{abstract}
This work demonstrates the use of phononic crystals (PnCs) to create solid-state phononic waveguides in standard CMOS technology. Such waveguides confine vibrational energy in CMOS frontend-of-line (FEOL) layers. Fully-differential driving ensures wave guiding and lowers scattering. Unreleased CMOS resonant body transistors (RBTs) based on these waveguides were implemented in IBM 32nm SOI technology. Quality factors exceeding 13,000 are achieved at $3.185 \mathrm{GHz}$ for an $f_{o} \cdot Q$ of $4.2 \times 10^{13}$, marking the highest $f_{o} \cdot Q$ RBT and highest $f_{o} \cdot Q$ unreleased MEMS resonator to date.
\end{abstract}

\section{INTRODUCTION}

High- $Q$ resonators are crucial components for filters and low phase noise oscillators, which are essential for RF, analog and digital circuits. With the ever expanding demands for higher communication data rates and fast data processing, it is desirable for resonators to scale to $\mathrm{GHz}$-frequencies and beyond. Wearable electronics and medical implants are examples of critical battery-operated systems that necessitate minimization of power consumption, size and cost. Micro-Electro-Mechanical (MEM) resonators are an attractive solution for today's demands, with $Q>10^{4}$ at GHz-frequencies and footprint on the order of $50 \mu^{2}$. Their monolithic integration with CMOS has been the subject of many research studies, with MEMS-first, MEMS-last and CMOS-MEMS being the most notable approaches [1].

The authors have previously demonstrated a new class of unreleased CMOS MEMS resonators, the Resonant Body Transistor (RBT), fabricated in the same process and side-by-side with standard CMOS transistors, without the need for any post-processing or packaging [2]. These RBTs rely on phononic crystals constructed in the CMOS back-end-of-line (BEOL) layers to achieve elastic energy confinement. Acoustic resonance is detected by active FET sensing, where the stress in the channel modulates the mobility of the sensing transistor [6]. MOS capacitors (MOSCAPs) are used for electrostatic actuation. It is also important to notice that scaling to $\mathrm{GHz}-$ frequencies has the advantage of making Landau-Rumer regime the dominant phonon relaxation process. This allows $f_{o} \cdot Q$ to scale linearly with $f_{o}$, enabling higher $Q$ devices.

In contrast, this work demonstrates phononic waveguides in standard CMOS technology, that confine the elastic vibrational energy vertically to the CMOS front-end-of-line (FEOL) layers. Confinement is achieved by reflection from the top by using a BEOL $\mathrm{PnC}$ as in [2], and from the bulk wafer by virtue of the faster sound velocity therein. This is a phenomenon similar to index guiding in photonic waveguides [3]. For CMOS RBTs, fully-differential driving is proven to be essential to ensure wave guiding and to minimize scattering. This wave guiding allows for $>50 \times$ increase in quality factor of unreleased CMOS MEMS resonators. RBTs based on these phononic waveguides with fully-differential drive and sense are implemented in IBM 32nm SOI technology as shown in Fig. 1.

\section{DEVICE DESIGN \\ BEOL Phononic Crystal}

PnCs are periodic structures composed of materials with contrasting acoustic impedance, which are characterized by the

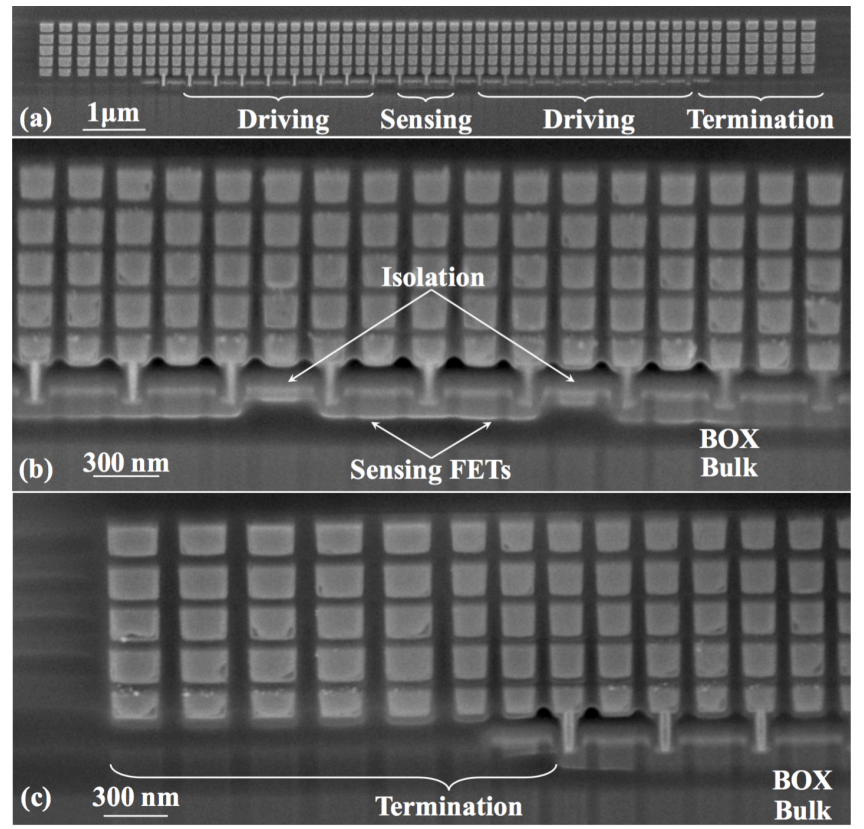

Figure 1: SEM micrograph showing (a) the full RBT structure (b) sensing FETs with isolation gates and (c) the termination waveguide section.

presence of bandgaps in their dispersion relation. Such bandgaps prohibit wave propagation in at least one direction, within the corresponding frequency range. Table 1 shows the mechanical properties of common CMOS BEOL materials. Large acoustic impedance contrast can be observed, especially between copper and the low-k $\mathrm{SiCOH}$ dielectric. This enables the realization of BEOL PnCs with large bandgaps. The latter are highly desirable to minimize the effect of CMOS process variations and to ensure large reflections with small number of PnC layers [2].

Table 1: Common CMOS BEOL material properties

\begin{tabular}{rccccc} 
Material & $\begin{array}{c}\rho \\
\left(\mathrm{kg} / \mathrm{m}^{3}\right)\end{array}$ & $\begin{array}{c}c_{11} \\
(\mathrm{GPa})\end{array}$ & $\begin{array}{c}Z_{11} \\
(\mathrm{MRayl})\end{array}$ & $\begin{array}{c}c_{44} \\
(\mathrm{GPa})\end{array}$ & $\begin{array}{c}Z_{44} \\
(\mathrm{MRayl})\end{array}$ \\
\hline $\mathrm{SiO} 2$ & 2200 & 75.2 & 12.9 & 29.9 & 8.1 \\
\hline Tungsten & 17600 & 525.5 & 96.2 & 160.5 & 53.1 \\
\hline SiCOH & 1060 & 3.96 & 2.05 & 1.32 & 1.18 \\
\hline Copper & 8700 & 176.5 & 39.1 & 40.7 & 18.8 \\
\hline
\end{tabular}

Fig. 2 shows an example of a BEOL PnC implemented in IBM $32 \mathrm{~nm}$ SOI technology using copper metal line in a background of $\mathrm{SiCOH}$ dielectric. This particular structure, with $154 \mathrm{~nm}$ metal line width spaced by $66 \mathrm{~nm}$, demonstrates a $3 \mathrm{GHz}(71 \%)$ complete bandgap. COMSOL FEM simulation with periodic boundary conditions was used to obtain the $\mathrm{PnC}$ dispersion relation. This $\mathrm{PnC}$ can be used as a strong reflector to provide good energy confinement in unreleased CMOS RBTs as demonstrated in [2]. While it provides high reflection from the top of the RBT cavity, it cannot prevent acoustic wave radiation into the CMOS die substrate [3]. A phononic waveguide must instead be implemented to achieve full vertical energy confinement. 


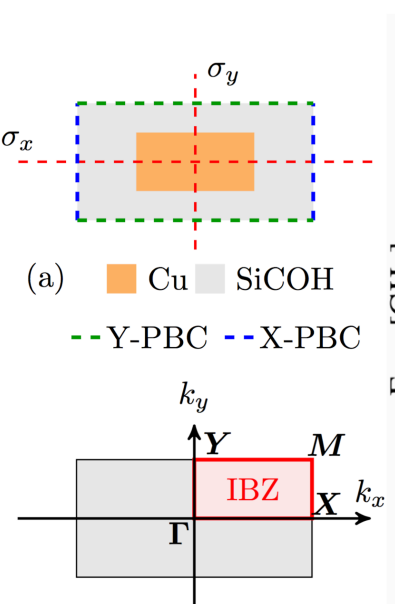

(b)

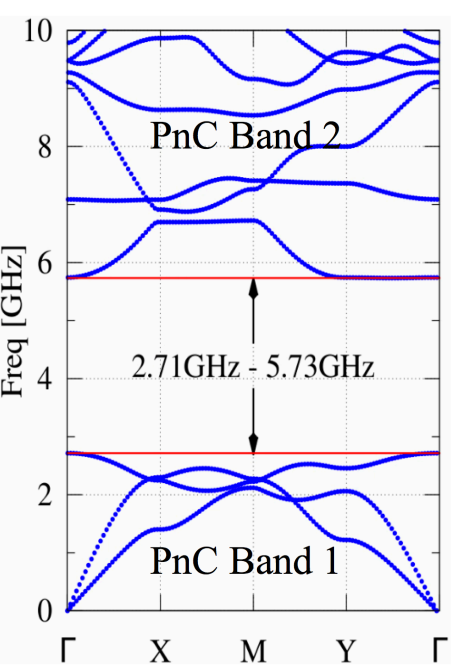

Figure 2: (a) Phononic Crystal (PnC) 2D unit cell using BEOL metals with periodic boundary conditions in $x$ and $y$. Cu metal width is $154 \mathrm{~nm}$ spaced by 66nm. (b) Reciprocal $k$-space and Irreducible Brillouin Zone (IBZ). (c) Dispersion relation showing $3 \mathrm{GHz}(71 \%)$ bandgap.

\section{Phononic Waveguides}

Considering the BEOL PnC of the previous section, on top of the FEOL layers and bulk wafer, it is possible to construct a phononic waveguide that can fully confine elastic waves to the CMOS FEOL layers. In particular, consider the structure of Fig. 3(a), where FEOL transistor gates are included at double the PnC horizontal period, together with the active area tungsten contacts. The resulting structure is periodic in the $x$-direction. The $x$-component $k_{x}$ of the wave vector $\vec{k}$ is conserved throughout the structure by virtue of the translational symmetry [3].

COMSOL FEM simulation of one horizontal period was used to obtain the dispersion relation of the phononic waveguide, with Floquet periodic boundary conditions (PBC) in $x$, parameterized by $k_{x}$. The resulting dispersion relation is shown in Fig. 3(b). Five types of modes can be observed in this dispersion relation. (i) $\mathrm{PnC}$ modes: these are modes corresponding to the PnC bands (band 1 and 2) that can propagate upwards in the PnC. (ii) Modes inside the PnC bandgap: these are evanescent waves that cannot propagate upwards in the PnC. These are not shown in the dispersion relation of Fig. 2(c) as they are not supported by infinitely periodic PnC structures. (iii) Bulk propagating modes: these are plane waves in the bulk wafer with dispersion relation $\omega=c|\vec{k}|$, or $\omega>c k_{x}$ for a given $k_{x}$, with $c$ the corresponding sound velocity. These modes form the continuum referred to as the sound cone in Fig. 3(b), which is bounded by the sound line $\omega=c k_{x}$. (iv) Evanescent modes in the bulk wafer: these are modes below the sound line with $k_{x}>\omega / c$, yielding an imaginary $k_{y}$ that is characteristic of evanescent modes. (v) Localized or guided modes: these modes are both inside the PnC bandgap and below the sound line. They are evanescent waves both in the $\mathrm{PnC}$ and the bulk wafer and can not propagate in either. These modes are vertically confined to the FEOL layers, creating a horizontal waveguide in the $x$-direction. Due to the energy confinement, the resulting spectrum is discrete as in Fig. 3(b). Such modes are ideal candidates for unreleased CMOS RBTs as they provide the necessary energy confinement to achieve a high- $Q$ resonator, as well as sufficient frequency separation for spurious mode reduction.

It is also important to note that the reflection achieved from the bulk wafer is mainly due to the higher sound velocity in the bulk silicon. This phenomenon is similar to index guiding in photonics (or total internal reflection from Snell's law). The SOI buried oxide

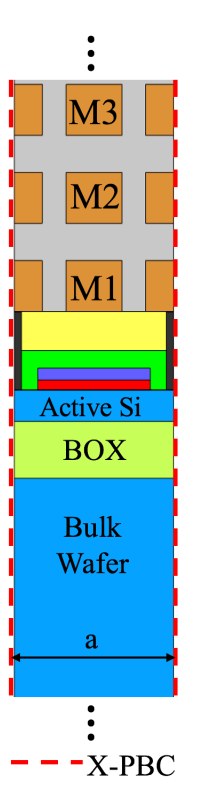

(a)

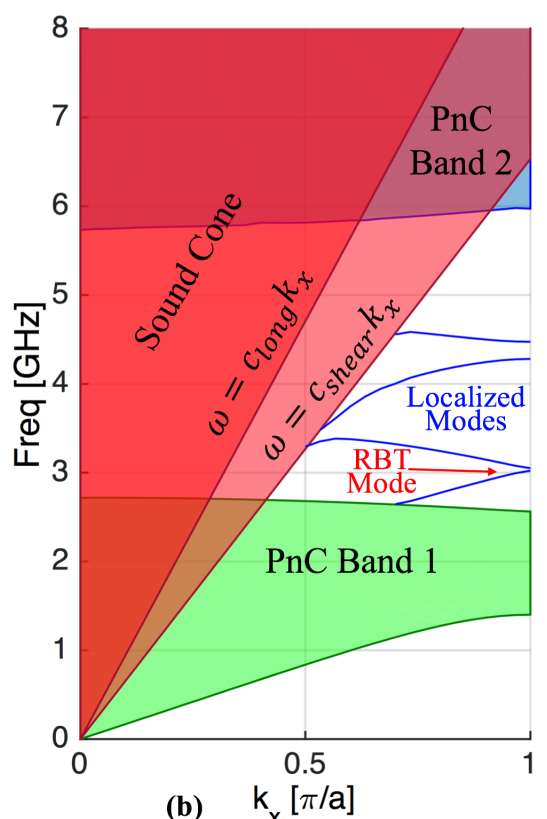

(b) $\mathrm{k}_{\mathrm{x}}[\pi / \mathrm{a}]$
Figure 3: (a)1D CMOS waveguide unit cell (one horizontal period) with the PnC of Fig. 2 and FEOL MOSCAP transducers (b) Dispersion relation for the horizontal waveguide showing vertically confined RBT modes.

(BOX) layer does not play any role in the energy confinement and this wave guiding is applicable in bulk CMOS technologies.

\section{Fully-Differential Driving}

While the proposed phononic waveguides offer a solution for elastic wave confinement in RBTs, wave guiding is restricted to a certain range of $k_{x}$ values. Fig. 3(b) indicates that for a given frequency $\omega$, wave guiding only happens for $k_{x} \in\left(\frac{\omega}{c}, \frac{\pi}{a}\right]$. RBTs must operate in this particular $k_{x}$ range.

Furthermore, one must consider the effect of perturbations in the design of the waveguide. Random perturbations arise from stochastic process variations, which are prominent in modern CMOS. Systematic perturbations arise when the waveguide characteristics are changed either from systematic process variations (e.g. overetching) or by design (e.g. to implement a termination). Perturbations to the waveguide structure will potentially result in scattering, which is the coupling of the main waveguide mode to other undesired modes. Scattering has adverse effects on the resonator: (i) in the case of scattering to a radiation mode, the scattering process represents energy losses that reduce the quality factor of the resonator; whereas (ii) scattering to guided modes can potentially lead to spurious modes appearing at different frequencies. As highlighted in [7], to a first order, scattering amplitude is inversely proportional to the difference in $k_{x}$ between the modes under consideration. Scattering at a given frequency corresponds to modes on a horizontal line in Fig. 3(b). For the guided modes, the sound line represents the nearest mode in $\vec{k}$-space.

To reduce the effect of scattering, it is important to operate as far as possible from the sound line, or at $k_{x}=\pi / a$. This also has the advantage of yielding the fastest evanescent decay for elastic waves in the bulk wafer, as the imaginary $y$-component of $\vec{k}$ is given by

$$
k_{y}=i \sqrt{\left(\frac{\pi}{a}\right)^{2}-\left(\frac{\omega}{c}\right)^{2}}
$$

Faster evanescent decay corresponds to higher stress at the driving and sensing transistor gates. 
(a)
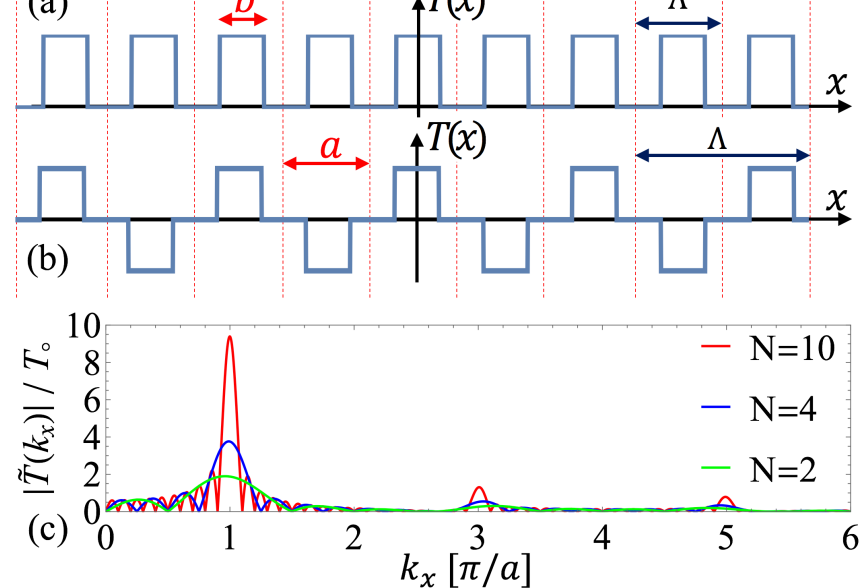

Figure 4: Spatial distribution of actuation stress for (a) in-phase and (b) fully-differential driving, along with (c) a comparison between the $k$-space components of fully-differential driving for finite driving transducer with 2,4 and 10 periods.

Specific $k_{x}$ values can be enforced by the spatial distribution of the RBT drive transducer. Operating at $k_{x}=\pi / a$ simply corresponds to neighboring periods of the waveguide being $180^{\circ}$ out-ofphase. This can be formally proven by considering the Fourier series expansion of the driving stress waveform in $\vec{k}$-space. Consider the in-phase driving of Fig. 4(a). The transducer has a period $\Lambda=a$, where the stress $T_{\text {。 }}$ is constant over the length $b$. Fourier series expansion for the stress is given by

$$
T(x)=\sum_{n} \frac{T_{\mathrm{o}} b}{a} \operatorname{sinc}\left(\frac{n \pi b}{a}\right) \exp \left(i \frac{2 \pi n}{a} x\right) .
$$

The components fall entirely in the sound cone at $k_{x}=$ $2 \pi n / a, \forall n \in \mathbb{N}$. This corresponds to plane wave radiation normal to the FEOL layers into the bulk wafer. On the other hand, the fullydifferential driving of Fig. 4(b) has a period $\Lambda=2 a$ and a Fourier series expansion given by

$$
T(x)=-\sum_{n} i^{3 n+1} T_{\circ} \operatorname{sinc}\left(\frac{n \pi}{2}\right) \sin \left(\frac{n \pi b}{2 a}\right) \exp \left(i \frac{\pi n}{a} x\right) .
$$

Fully-differential driving shows components at $k_{x}=n \pi / a, \forall n \in$ $\mathbb{N}$, which are non-trivial only for odd $n$. This can be intuitively explained by considering the radiation field in the bulk wafer far away from the driving transducers. At a distance $l \gg a$ in the bulk, outof-phase radiated fields from neighboring transducers destructively interfere together, resulting in a negligible radiation field.

The above analysis is for ideal infinitely periodic transducers. However, real resonators incorporate finite transducers. Fourier transform should be used instead of Fourier series and a continuous spectrum is obtained in $\vec{k}$-space. The larger the number of the transducer periods the narrower the spectrum in $\vec{k}$-space will be, which helps in lowering scattering. This is highlighted in Fig. 4(c), where the spectrum of finite transducers is compared for different number of fully-differential periods $(\Lambda=2 a)$. Scattering reduction favors a large number of driving periods, however, this comes at the cost of resonator footprint, input capacitance, as well as increased random process variations. This represents a design trade-off.

\section{Full RBT Structure}

An RBT based on phononic waveguides is implemented in IBM $32 \mathrm{~nm}$ SOI technology. The full RBT structure is shown in Fig. 5. Two MOSFETs are used for fully-differential active sensing in the middle of the RBT structure. Eight MOSCAPs (driven $180^{\circ}$ out-ofphase) are used for fully-differential electrostatic internal dielectric drive on each side. This enforces the condition $k_{x}=\pi / a$ for

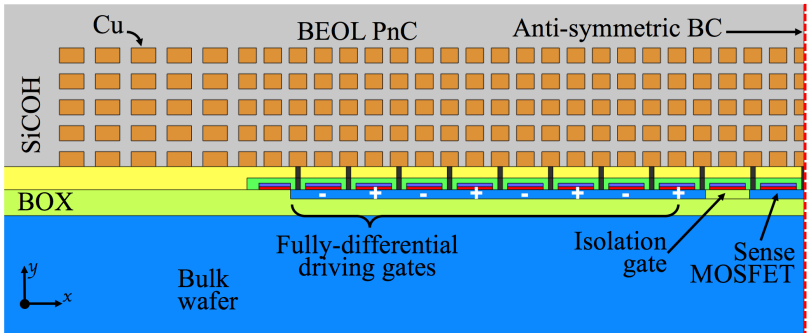

Figure 5: Cross section of the vertically confined RBT design (half structure) with differential capacitive actuation and transistor sensing.

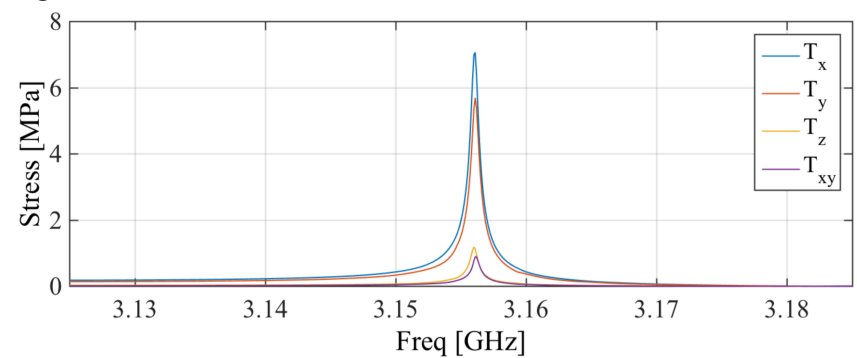

Figure 6: Simulated stresses at sensing FET in the resonator assuming bulk material properties.

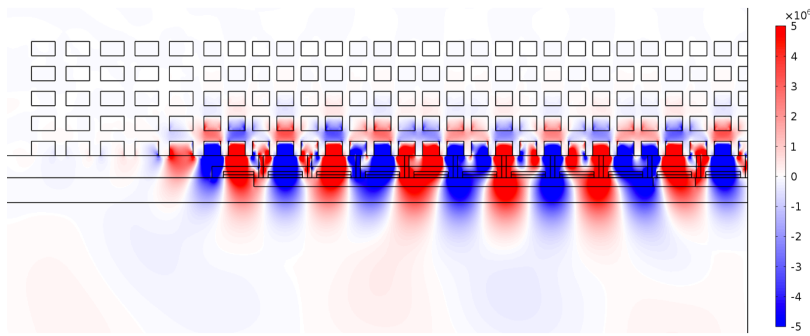

Figure 7: Simulated y-stress of the waveguided RBT mode at $3.155 \mathrm{GHz}$ resonance.

reduced scattering with sufficient mode uniformity, while maintaining relatively small resonator footprint. Phononic waveguides with mismatched characteristics have been used at both ends to terminate the cavity. Shallow-trench isolation (STI) is used to separate the driving MOSCAPs from the sensing MOSFETs for better electrical isolation. As seen in Fig. 5, the STI isolation in this technology is sufficiently shallow, causing small perturbation in the waveguide structure. However, numerical optimization for the full RBT structure is required to match the different waveguide sections and avoid scattering [8]. The SOI BOX layer further contributes to the electrical isolation.

FEM frequency domain simulation is used to assess the performance of the fully-optimized RBT. The average stresses along the channel length of the sense MOSFET are shown in Fig. 6. The ystress for the resonance mode at $3.155 \mathrm{GHz}$ is shown in Fig. 7. The mode is confined to the FEOL layers, with evanescent decay in the bulk wafer, BEOL PnC, as well as the termination.

\section{EXPERIMENTAL RESULTS}

Waveguided RBTs in this work were fabricated in IBM $32 \mathrm{~nm}$ SOI technology. SEM micrographs of the RBT are shown in Fig. 1, highlighting the phononic crystal, sensing FETs, as well as the termination waveguides. Fabricated resonators occupy an area of $4 \mu \mathrm{m} \times 13 \mu \mathrm{m}$.

Fig. 8 shows an optical micrograph of the RBT probing pads, along with the DC biasing connection. Fully-differential RF GSSG 


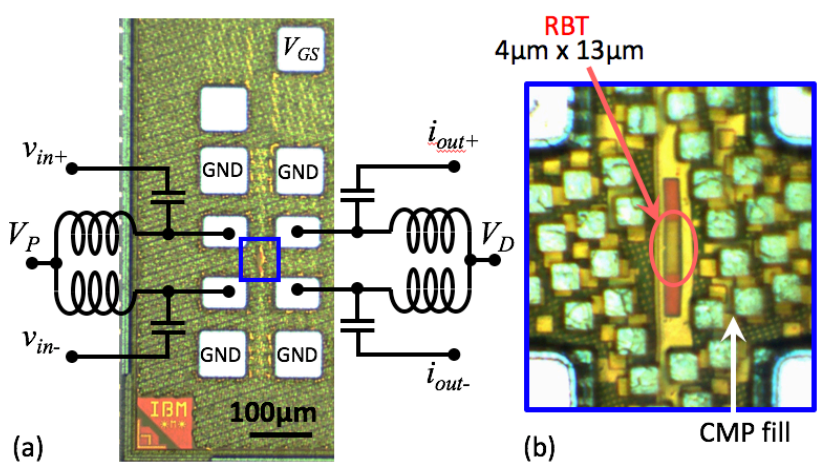

(a)

(b)

Figure 8: (a) Optical micrograph of the resonator showing probing pads and (b) zoomed-in top-view of the RBT.

probes were used for driving and sensing ports of the RBT. The driving MOSCAPs was biased in strong inversion with $V_{p}=1 \mathrm{~V}$. The sensing FETs were biased in linear regime with $V_{g s}=$ $0.7 \mathrm{~V}$ and $V_{d s}=85 \mathrm{mV}$ to maximize the sensitivity of the drain current to channel mobility modulation. Drain current for a single sensing FET was $115 \mu \mathrm{A}$. RF testing power was limited to $-20 \mathrm{dBm}$, which corresponds to a peak voltage of $60 \mathrm{mV}$ on the MOSCAPs, in order not to perturb the DC operating point.

An Agilent N5225A PNA was used for RF characterization, which was performed at room temperature in air. The front-panel couplers on the PNA were reversed for a $15 \mathrm{~dB}$ enhancement in sensitivity. As a result, the PNA transmitted power was reduced by the same factor. To compensate for this loss, the testing power was increased to $-5 \mathrm{dBm}$. Full 4-port S-parameters measurements were performed for the RBTs. On-chip open structures were used for deembedding. An electromechanical transconductance $g_{e m}$ was extracted from the resulting Y-parameters as $g_{e m}=Y_{21}-Y_{12}$, according to the standard transistor $\pi$-model. An IF bandwidth of $3 \mathrm{kHz}$, along with 30-point average were used. A dwell time of $20 \mu$ s was selected to allow for resonance build-up. Additionally, a smoothing filter with a 9-point aperture was used to reduce the peak-to-peak noise.

The measured fully-differential $g_{\text {em }}$ of one RBT is shown in Fig. 9, demonstrating resonance at $3.1885 \mathrm{GHz}$ with a FWHM quality factor $Q \sim 13,300$ for an $f_{o} \cdot Q$ of $4.2 \times 10^{13}$. This marks the highest $\mathrm{f}_{\mathrm{o}} \cdot Q \mathrm{RBT}$ and highest $f_{o} \cdot Q$ unreleased MEMS resonator to date.

\section{CONCLUSION}

Phononic crystals in CMOS BEOL layers were used to realize unreleased phononic waveguides that confine elastic vibrations in the CMOS FEOL layers. Reflections from the bulk substrate are achieved by a phenomenon similar to index-guiding in photonics. Such phononic waveguides can be used as the basis for a resonance cavity of unreleased CMOS RBTs, mechanical coupling thereof, or phononic networks for advanced signal processing. Fully-differential driving is essential for wave guiding and scattering reduction.

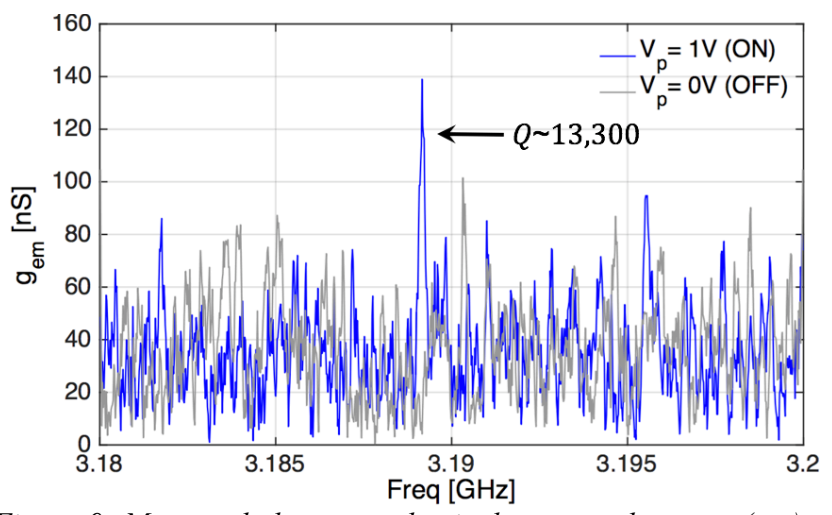

Figure 9: Measured electro-mechanical transconductance $\left(g_{\text {em }}\right)$ of the $R B T$, with record breaking $f_{o} \cdot Q$, in close agreement with simulation.

RBTs implemented in IBM $32 \mathrm{~nm}$ SOI achieve $Q \sim 13,300$ at $3.19 \mathrm{GHz}$, for an $f_{o} \cdot Q$ of $4.2 \times 10^{13}$, marking $56 \times$ increase over previous design. The solid-state nature of these devices and small feature sizes available in advanced CMOS nodes enable high- $Q \mathrm{GHz}$-frequency operation, with relaxed packaging and operating voltage constraints.

\section{REFERENCES}

[1] G.K. Fedder et al., "Technologies for cofabricating MEMS and electronics". Proceedings of the IEEE, 96(2), Feb 2008.

[2] B. Bahr, R. Marathe, D. Weinstein, "Theory and Design of Phononic Crystals for Unreleased CMOS-MEMS Resonant Body Transistors," IEEE JMEMS, vol.24, no.5, Oct. 2015

[3] J. D. Joannopoulos, S. G. Johnson, et. al., "Photonic Crystals: Molding the Flow of Light," $2^{\text {nd }}$ ed. Princeton Univ. Press, 2011.

[4] D. Weinstein and S. A. Bhave, "Acoustic resonance in an Independent-Gate FinFET," Solid State Sensor, Actuator and Microsystems Workshop (Hilton Head 2010), pp. 459-462.

[5] S. G. Johnson et al., "Coupling, scattering, and perturbation theory: Semi-analytical analyses of photonic-crystal waveguides," 5th Int. Conf. Transparent Opt. Netw., vol. 1. Jun./Jul. 2003, pp. 103-109.

[6] D. Weinstein and S. Bhave, "The resonant body transistor", Nano Letters, 2010, pp 1234-1237

[7] S. G. Johnson, P. Bienstman, M. A. Skorobogatiy, M. Ibanescu, E. Lidorikis, and J. D. Joannopoulos, "Adiabatic theorem and continuous coupled-mode theory for efficient taper transitions in photonic crystals," Phys. Rev. E, vol. 66, Dec. 2002.

[8] B. Bahr, L. Daniel and D. Weinstein, "Optimization of Unreleased CMOS-MEMS RBTs," accepted for publication in International Frequency Control Symposium (IFCS) 2016, IEEE.

\section{CONTACT}

*B. Bahr, tel: 617-452-2861; bichoy@mit.edu 